\title{
SUBORDINATION THEORY FOR HOLOMORPHIC MAPPINGS OF SEVERAL COMPLEX VARIABLES
}

\author{
GABRIELA KOHR and MIRELA KOHR-ILE \\ Faculty of Mathematics, Babeş-Bolyai University \\ R-3400 Cluj-Napoca, Romania
}

\begin{abstract}
The authors obtain a generalization of Jack-Miller-Mocanu's lemma and, using the technique of subordinations, deduce some properties of holomorphic mappings from the unit polydisc in $\mathbb{C}^{n}$ into $\mathbb{C}^{n}$.
\end{abstract}

1. Introduction. Let $n$ be a positive integer and $\mathbb{C}^{n}$ denote the space of $n$ complex variables $z=\left(z_{1}, \ldots, z_{n}\right)$ with the Euclidean product $\langle z, w\rangle=\sum_{k=1}^{n} z_{k} \bar{w}_{k}$ and the Euclidean norm $|z|=\langle z, z\rangle^{1 / 2}$. Let $U_{1}^{n}$ denote the unit polydisc in $\mathbb{C}^{n}$, i.e. the set $\left\{z \in \mathbb{C}^{n}:\|z\|<1\right\}$, where $\|z\|=\max _{1 \leq j \leq n}\left|z_{j}\right|$, and let $B_{1}^{n}$ stand for the open unit Euclidean ball in $\mathbb{C}^{n}$. For $n=1, B_{1}^{n}=U_{1}^{n}=U=\{z \in \mathbb{C}:|z|<1\}$, the unit disc in $\mathbb{C}$.

Recently the present authors [3] have obtained a new generalization of the Jack-MillerMocanu lemma and, using the technique of subordinations, arrived at some properties of holomorphic mappings defined on the unit polydisc $U_{1}^{n}$. In this paper one deduces other results concerning partial differential subordinations and some inequalities for holomorphic mappings on $U_{1}^{n}$.

Let $\Omega$ be a domain in $\mathbb{C}^{n}$ and let $H(\Omega)$ be the set of holomorphic mappings on $\Omega$. If $f \in H(\Omega)$, denote by $[D f(z)], z \in \Omega$, its Fréchet matrix $\left[\left(\partial / \partial z_{j}\right) f_{k}(z)\right]_{j, k=1, \ldots, n}$ and by $[D f(z)]^{\prime}$ its transpose. Also, if $F$ is a holomorphic function defined on a domain $D \subseteq \mathbb{C}^{n}$, then by $(\partial / \partial z) F$ we denote the complex vector $\left(\left(\partial / \partial z_{1}\right) F, \ldots,\left(\partial / \partial z_{n}\right) F\right)$ and by $[(\partial / \partial z) F]^{\prime}$ its transpose. (If $z \in \mathbb{C}^{n}$, then $[z]^{\prime}$ means the transpose of $z$.) Since $\left(\mathbb{C}^{n},|\cdot|\right)$ is a normed space with respect to the Euclidean norm, if $A: \mathbb{C}^{n} \rightarrow \mathbb{C}^{n}$ is a continuous and linear operator, then by $|A|$ we denote the norm of $A$, i.e., $|A|=\sup _{|u|=1}|A u|$. For our purpose we shall use the following result.

LEMma 1.1 [2]. Let $0<r_{0}<1$ and $h: r_{o} \bar{U}_{1}^{n} \rightarrow \mathbb{C}$ be a holomorphic function on $r_{o} \bar{U}_{1}^{n}$ with $h(0)=0$. If $z_{0} \in r_{o} \bar{U}_{1}^{n}$ and $\left|h\left(z_{0}\right)\right|=\max \left\{|h(z)|: z \in r_{o} \bar{U}_{1}^{n}\right\}$, then at $z=z_{0}$ we

1991 Mathematics Subject Classification: Primary 32A10; Secondary 32H99.

The paper is in final form and no version of it will be published elsewhere. 
have

$$
z_{0}\left[\frac{\partial h\left(z_{0}\right)}{\partial z}\right]^{\prime}=m h\left(z_{0}\right) \quad \text { and } \quad \operatorname{Re}\left\{\frac{z_{0}}{h\left(z_{0}\right)} \frac{\partial^{2} h\left(z_{0}\right)}{\partial z^{2}}\left[z_{0}\right]^{\prime}\right\} \geq m(m-1), \quad \text { where } \quad m \geq 1 .
$$

2. Main results. We start with

THEOREM 2.1. Let $f: U_{1}^{n} \rightarrow \mathbb{C}^{n}$ be a holomorphic mapping on $U_{1}^{n}$ such that $f(0)=0$ and $f(z) \not \equiv 0, z \in U_{1}^{n}$. Let $0<r_{0}<1$ and $z_{0} \in r_{o} \bar{U}_{1}^{n}$ be such that

$$
\left|f\left(z_{0}\right)\right|=\max \left\{|f(z)|: z \in r_{o} \bar{U}_{1}^{n}\right\} .
$$

Then there exist real numbers $m$ and $s$ such that $s \geq m \geq 1$, and at $z=z_{0}$ we have

(i) $\left\langle\left[D f\left(z_{0}\right)\right]\left[z_{0}\right]^{\prime}, f\left(z_{0}\right)\right\rangle=m\left|f\left(z_{0}\right)\right|^{2}$ and

(ii) $\left\langle\left|\left[D f\left(z_{0}\right)\right]\left[z_{0}\right]^{\prime}\right|=s\left|f\left(z_{0}\right)\right|\right.$.

Proof. Using the hypothesis we can assume $z_{0} \neq 0$ and $f\left(z_{0}\right) \neq 0$. Let $g: U_{1}^{n} \rightarrow \mathbb{C}$, be defined by $g(z)=\left\langle f(z), f\left(z_{0}\right)\right\rangle, z \in U_{1}^{n}$; then $g$ is holomorphic on $U_{1}^{n}, g(0)=0$ and $g$ satisfies $\left|g\left(z_{0}\right)\right|=\max \left\{|g(z)|:|| z|| \leq r_{0}\right\}$. From Lemma 1.1 we deduce that there exists $m \in \mathbb{R}, m \geq 1$ such that $z_{0}\left[(\partial / \partial z) g\left(z_{0}\right)\right]^{\prime}=m g\left(z_{0}\right)$. Yet,

$$
z_{0}\left[\frac{\partial g\left(z_{0}\right)}{\partial z}\right]^{\prime}=\sum_{k=1}^{n} z_{0}^{k} \frac{\partial g\left(z_{0}\right)}{\partial z_{k}}=\sum_{k=1}^{n} z_{0}^{k}\left[\sum_{j=1}^{n} \overline{f_{j}\left(z_{0}\right)} \frac{\partial f_{j}\left(z_{0}\right)}{\partial z_{k}}\right]=\left\langle\left[D f\left(z_{0}\right)\right]\left[z_{0}\right]^{\prime}, f\left(z_{0}\right)\right\rangle .
$$

Hence we obtain (i). On the other hand, $\left|\left\langle\left[D f\left(z_{0}\right)\right]\left[z_{0}\right]^{\prime}, f\left(z_{0}\right)\right\rangle\right| \leq\left|f\left(z_{0}\right)\right|\left|\left[D f\left(z_{0}\right)\right]\left[z_{0}\right]^{\prime}\right|$, so from (i) we have $\left|\left[D f\left(z_{0}\right)\right]\left[z_{0}\right]^{\prime}\right| \geq m\left|f\left(z_{0}\right)\right|$, which implies that there exists $s \in \mathbb{R}$, $s \geq m$, such that $\left|\left[D f\left(z_{0}\right)\right]\left[z_{0}\right]^{\prime}\right|=s\left|f\left(z_{0}\right)\right|$.

Remark 2.1. For $n=1$ we obtain the result of Jack-Miller-Mocanu's lemma [5], $[6]$.

Let $M$ and $s$ be real numbers such that $M>0$ and $s \geq 1$. Let further $D \subseteq \mathbb{C}^{2 n}$ be a domain such that $(0,0) \in D$.

Definition 2.1. Let $K_{n}=\cup_{s \geq 1} K_{n}^{s}(M)$, where $K_{n}^{s}(M)=\left\{(u, v) \in \mathbb{C}^{2 n}:|u|=\right.$ $M,|v|=s M\}$. Suppose that $K_{n} \subset D$ and let $V_{n}(D, M)=\left\{g: D \rightarrow \mathbb{C}^{n}: g\right.$ is continuous on $D,|g(0,0)|<M,|g(u, v)| \geq M$, for all $\left.(u, v) \in K_{n}\right\}$.

By using this definition and the result of Theorem 2.1, we deduce

THEOREM 2.2. Let $D \subseteq \mathbb{C}^{2 n}$ be a domain and $f$ be a holomorphic mapping from $U_{1}^{n}$ into $\mathbb{C}^{n}$ such that $f(0)=0$ and $f(z) \not \equiv 0, z \in U_{1}^{n}$. Suppose there exists $g \in V_{n}(D, M)$ such that

$$
\left(f(z),[D f(z)][z]^{\prime}\right) \in D \quad \text { and } \quad\left|g\left(f(z),[D f(z)][z]^{\prime}\right)\right|<M \quad \text { for all } \quad z \in U_{1}^{n} .
$$

Then $|f(z)|<M, z \in U_{1}^{n}$.

Proof. If the relation $|f(z)|<M$ does not hold everywhere in $U_{1}^{n}$, then, using the continuity of the norm and $f(0)=0$, we deduce that there exists $z_{0} \in r_{0} \bar{U}_{1}^{n}, 0<r_{0}<1$, and $M=\left|f\left(z_{0}\right)\right|=\max \left\{|f(z)|:\|z\| \leq r_{0}\right\}$. Then by Theorem 2.1 there exists $s \in \mathbb{R}$, $s \geq 1$, such that at $z=z_{0}$ we have $\left|\left[D f\left(z_{0}\right)\right]\left[z_{0}\right]^{\prime}\right|=s\left|f\left(z_{0}\right)\right|$. If we set $u=f\left(z_{0}\right)$ and 
$v=\left[D f\left(z_{0}\right)\right]\left[z_{0}\right]^{\prime}$, then $(u, v) \in K_{n}^{s}(M)$. Hence, by $g \in V_{n}(D, M)$, we have $|g(u, v)| \geq M$, so we obtain a contradiction with the hypothesis. Therefore $|f(z)|<M$ for all $z \in U_{1}^{n}$.

Rem ark 2.2. It is interesting that this result can be applied for proving that some partial differential equations in $\mathbb{C}^{n}$ have bounded solutions.

Corollary 2.1. Let $F: U_{1}^{n} \rightarrow \mathbb{C}^{n}$ be a holomorphic mapping on $U_{1}^{n}$, which satisfies $F(0)=0$ and $|F(z)|<M$ for all $z \in U_{1}^{n}$. Let $g \in V_{n}(D, M)$ be a holomorphic mapping and suppose that the differential equation $g\left(f(z),[D f(z)][z]^{\prime}\right)=F(z), f(0)=0$, has on $U_{1}^{n}$ a holomorphic solution $f$. Then $|f(z)|<M$ for all $z \in U_{1}^{n}$.

Definition 2.2. Let $\omega: U_{1}^{n} \rightarrow \mathbb{C}^{n}$ be a holomorphic mapping on $U_{1}^{n}$. We say that $\omega$ is a Schwarz mapping if $\omega(0)=0$ and $|\omega(z)|<1$ for all $z \in U_{1}^{n}$.

Definition 2.3. Let $f$ be a holomorphic mapping from $U_{1}^{n}$ into $\mathbb{C}^{n}$ and $g$ be a holomorphic mapping from $B_{1}^{n}$ into $\mathbb{C}^{n}$. We say that $f$ is subordinate to $g(f \prec g)$ if there exists a Schwarz mapping $\omega$ (in the sense of Definition 2.2) such that $f=g \circ \omega$ in $U_{1}^{n}$.

Remark 2.3. If $f$ is subordinate to $g$, then $f(0)=g(0)$ and $f\left(U_{1}^{n}\right) \subseteq g\left(B_{1}^{n}\right)$. Yet, if $g$ is biholomorphic on $B_{1}^{n}$, then we can easily show that $f \prec g$ if and only if $f(0)=g(0)$ and $f\left(U_{1}^{n}\right) \subseteq g\left(B_{1}^{n}\right)$. Also, if $f \prec g$, then $f\left(r \bar{U}_{1}^{n}\right) \subseteq g\left(r \bar{B}_{1}^{n}\right)$ for all $0<r<1$.

Let $Q_{n}$ be denote the family of all biholomorphic mappings $g$ on $\bar{B}_{1}^{n} \backslash E(g)$, where

$$
E(g)=\left\{\zeta \in \partial B_{1}^{n}: \text { there exists } k, 1 \leq k \leq n, \text { with } \lim _{\substack{|z|<1 \\ z \rightarrow \zeta}} g_{k}(z)=\infty\right\} .
$$

In the next (except for some examples) we shall suppose that $E(g)=\emptyset$; in the other case we can use in the proofs the class $Q_{n}$.

Now we can give the following result:

THEOREM 2.3. Let $f$ be a holomorphic mapping on $U_{1}^{n}$ and let $g$ be a biholomorphic mapping on $\bar{B}_{1}^{n}$ such that $f(0)=g(0)$. If $f$ is not subordinate to $g$, then there exist real numbers $m$ and $s, s \geq m \geq 1$, and points $z_{0} \in U_{1}^{n}, 0<\left\|z_{0}\right\|<1, \zeta_{0} \in \partial B_{1}^{n}$, such that

(i) $f\left(z_{0}\right)=g\left(\zeta_{0}\right), f\left(\left\{z:\|z\|<\left\|z_{0}\right\|\right\}\right) \subset g\left(B_{1}^{n}\right)$

and at $z=z_{0}$ we have

(ii) $\sum_{k=1}^{n} \bar{\zeta}_{0}^{k} \cdot z_{0}\left[D f\left(z_{0}\right)\right]^{\prime}\left[(\partial / \partial w) \tilde{g}_{k}\left(w_{0}\right)\right]^{\prime}=m$,

(iii) $s\left|\left[D g\left(\zeta_{0}\right)\right]^{-1}\right|^{-1} \leq\left|\left[D f\left(z_{0}\right)\right]\left[z_{0}\right]^{\prime}\right| \leq s\left|\left[D g\left(\zeta_{0}\right)\right]\right|$,

where $\zeta_{0}=\left(\zeta_{0}^{1}, \ldots, \zeta_{0}^{n}\right), w_{0}=g\left(\zeta_{0}\right), g^{-1}\left(w_{0}\right)=\left(\tilde{g}_{1}\left(w_{0}\right), \ldots, \tilde{g}_{n}\left(w_{0}\right)\right)$.

Proof. Since $f$ is not subordinate to $g$ and $f(0)=g(0)$, then $f\left(U_{1}^{n}\right) \nsubseteq g\left(B_{1}^{n}\right)$. Hence there exist $z_{0} \in U_{1}^{n},\left\|z_{0}\right\|=r_{0}, 0<r_{0}<1$ and $\zeta_{0} \in \partial B_{1}^{n}$ such that $f\left(z_{0}\right)=$ $g\left(\zeta_{0}\right)$ and $f\left(\left\{z:\|z\|<\left\|z_{0}\right\|\right\}\right) \subset g\left(B_{1}^{n}\right)$. Let $h: r_{0} \bar{U}_{1}^{n} \rightarrow \mathbb{C}^{n}$ be the mapping given by $h(z)=\left(g^{-1} \circ f\right)(z), z \in r_{0} U_{1}^{n}$. Then $h$ is holomorphic on $r_{0} U_{1}^{n}, h(0)=0$ and $1=\left|h\left(z_{0}\right)\right|=\max \left\{|h(z)|:\|z\| \leq r_{0}\right\}$. By applying the result of Theorem 2.1 and the continuity and linearity of the operators $D g\left(\zeta_{0}\right)$ and $\left[D g\left(\zeta_{0}\right)\right]^{-1}$ on $\left(\mathbb{C}^{n},|\cdot|\right)$, we obtain (ii) and (iii), as desired.

For $n=1$ we deduce 
Corollary $2.2[5,6]$. Let $f$ and $g$ be holomorphic functions on $U$ and $g$ be univalent on $\bar{U}$, such that $f(0)=g(0)$. If $f$ is not subordinate to $g$, then there exist $z_{0} \in U, \zeta_{0} \in \partial U$ and $m \geq 1$ such that $f\left(z_{0}\right)=g\left(\zeta_{0}\right)$ and $z_{0} f^{\prime}\left(z_{0}\right)=m \zeta_{0} g^{\prime}\left(\zeta_{0}\right)$.

Now, using the above results, we are able to introduce the concept of "admissible class" for mappings of several variables.

Definition 2.4. Let $D \subseteq \mathbb{C}^{n}, \Omega \subseteq \mathbb{C}^{2 n}$ be domains, $n \geq 1$, let $g$ be a biholomorphic mapping on $\bar{B}_{1}^{n}$, and $\zeta_{0} \in \bar{\partial} B_{1}^{n}$. Suppose that $H_{n}^{s}\left(\zeta_{0}, g\right)=\left\{(u, v) \in \mathbb{C}^{2 n}: u=g\left(\zeta_{0}\right)\right.$, $\left.s\left|\left[D g\left(\zeta_{o}\right)\right]^{-1}\right|^{-1} \leq|v| \leq s\left|\left[D g\left(\zeta_{0}\right)\right]\right|\right\}$, where $s, s \geq 1$, is a real number. Let further

$$
H_{n}(g)=\bigcup_{\substack{\left|\zeta_{0}\right|=1 \\ s \geq 1}} H_{n}^{s}\left(\zeta_{0}, g\right)
$$

and suppose $H_{n}(g) \subset \Omega$ and $(g(0), 0) \in \Omega$. The admissible class $\psi_{n}^{n}(\Omega, D, g)$ consists of those mappings $\psi_{n}: \Omega \times U_{1}^{n} \rightarrow \mathbb{C}^{n}$ which are continuous and satisfy $\psi_{n}(g(0), 0 ; 0) \in D$ and $\psi_{n}(v, v ; z) \notin D$ for all $(u, v) \in H_{n}(g)$ and $z \in U_{1}^{n}$.

Using the conclusion of Theorem 2.3 and the above definition we obtain:

THEOREM 2.4. Let $f$ be a holomorphic mapping on $U_{1}^{n}$ and let $g$ be a biholomorphic mapping on $\bar{B}_{1}^{n}$, such that $f(0)=g(0)$. Suppose that there exists $\psi_{n}^{n}(\Omega, D ; g)$ such that

$$
\left(f(z),[D f(z)][z]^{\prime}\right) \in \Omega \quad \text { and } \quad \psi_{n}\left(f(z),[D f(z)][z]^{\prime}\right) \in D \quad \text { for all } \quad z \in U_{1}^{n} .
$$

Then $f$ is subordinate to $g$.

Proof. If the subordination $f \prec g$ does not hold, then, by Theorem 2.3 , there exist $z_{0} \in U_{1}^{n}, \zeta_{0} \in \partial B_{1}^{n}$ and $s \in \mathbb{R}, s \geq 1$, such that $f\left(z_{0}\right)=g\left(\zeta_{0}\right)$ and the relations (ii) and (iii) hold. Yet, if we define $u=f\left(z_{0}\right)$ and $v=\left[D f\left(z_{0}\right)\right]\left[z_{0}\right]^{\prime}$, then $(u, v) \in H_{n}^{s}\left(\zeta_{0}, g\right) \subseteq H_{n}(g)$. Hence, from Definition 2.4, we deduce $\psi_{n}\left(u, v ; z_{0}\right) \notin D$ which is a contradiction with the hypothesis.

3. Examples. In this section we point out the usefulness of the above results.

Let $z \in \mathbb{C}^{n}, z=\left(z_{1}, \ldots, z_{n}\right)$; then we say that $\operatorname{Re} z \geq 0$ (resp. Re $z>0$ ) if and only if Re $z_{k} \geq 0$ (resp. Re $z_{k}>0$ ) for all $k \in\{1, \ldots, n\}$. Let $\tilde{1}=(1, \ldots, 1) \in \mathbb{C}^{n}$. Consider the mapping $g: U_{1}^{n} \rightarrow \mathbb{C}^{n}$, given by

$$
g(z)=\left(\frac{1+z_{1}}{1-z_{1}}, \ldots, \frac{1+z_{n}}{1-z_{n}}\right) \text { for all } z \in U_{1}^{n} .
$$

Then it is clear that $g$ is univalent on $U_{1}^{n}$ and $g\left(U_{1}^{n}\right)=E_{n}$, where $E_{n}=\left\{w \in \mathbb{C}^{n}\right.$ : Re $w>0\}$. Now, let $A=\left\{z \in \partial B_{1}^{n}\right.$ : there exists $k, 1 \leq k \leq n$ such that $\left.z_{k}=1\right\}$. In this case $E(g)=A$.

Moreover, we denote by $G_{n}^{s}\left(\zeta_{0}, \tilde{1}\right)$ the class

$$
G_{n}^{s}\left(\zeta_{0}, \tilde{1}\right)=\left\{(u, v) \in \mathbb{C}^{2 n}: u=\left(\frac{1+\zeta_{0}^{1}}{1-\zeta_{0}^{1}}, \ldots, \frac{1+\zeta_{0}^{n}}{1-\zeta_{0}^{n}}\right), \quad|v| \geq \frac{1}{2} s\right\}
$$

where $\zeta_{0}\left(\zeta_{0}^{1}, \ldots, \zeta_{0}^{n}\right) \in \partial B_{1}^{n} \backslash A$ and $s \geq 1$. Let $G_{n}(\tilde{1})=\bigcup_{s \geq 1}\left\{G_{n}^{s}\left(\zeta_{0}, \tilde{1}\right): \zeta_{0} \in \partial B_{1}^{n} \backslash A\right\}$. Let further $\psi_{n}^{n}(\tilde{1})$ be the class of those continuous mappings $\psi_{n}: \Omega \times U_{1}^{n} \rightarrow \mathbb{C}^{n}$, which 
satisfy $\psi_{n}(\tilde{1}, 0 ; 0) \in D$ and $\psi_{n}(u, v ; z) \notin D$, for all $z \in U_{1}^{n}$ and $(u, v) \in G_{n}(\tilde{1})$, where $\Omega \subseteq \mathbb{C}^{2 n}$ with $G_{n}(\tilde{1}) \subset \Omega$.

With the above notation we obtain

Theorem 3.1. Let $\Omega$ and $D$ be domains in $\mathbb{C}^{2 n}$ and $\mathbb{C}^{n}$, respectively, and $f \in H\left(U_{1}^{n}\right)$, $f(0)=\tilde{1}$. Suppose that there exists $\psi_{n} \in \psi_{n}^{n}(\tilde{1})$ such that

$$
\left(f(z),[D f(z)][z]^{\prime}\right) \in \Omega \quad \text { and } \quad \psi_{n}\left(f(z),[D f(z)][z]^{\prime} ; z\right) \in D \quad \text { for all } z \in U_{1}^{n} .
$$

Then $\operatorname{Re} f(z)>0$ in $U_{1}^{n}$.

Pro of. It is clear that if we prove $f \prec g$, where $g(z)$ is given by (2), then $\operatorname{Re} f(z)>0$, $z \in U_{1}^{n}$. If this subordination does not hold, then using the same reasons as in the proof of Theorem 2.3, we deduce that there exist points $z_{0} \in U_{1}^{n}, \zeta_{0} \in \partial B_{1}^{n} \backslash A$ such that

$$
f\left(z_{0}\right)=\left(\frac{1+\zeta_{0}^{1}}{1-\zeta_{0}^{1}}, \ldots, \frac{1+\zeta_{0}^{n}}{1-\zeta_{0}^{n}}\right) \quad \text { and } \quad\left|\left[D f\left(z_{0}\right)\right]\left[z_{0}\right]^{\prime}\right| \geq \frac{1}{2} s, \quad s \geq 1 .
$$

Let $u=f\left(z_{0}\right)$ and $v=\left[D f\left(z_{0}\right)\right]\left[z_{0}\right]^{\prime}$; then it is clear that $(u, v) \in G_{n}^{s}\left(\zeta_{0}, \tilde{1}\right)$, so using the definition of the class $\psi_{n}^{n}(\tilde{1})$ we conclude that $\psi_{n}\left(u, v ; z_{0}\right) \notin D$, but this contradicts the hypothesis. Therefore $f$ is subordinate to $g$, as desired.

An immediate application of Theorem 2.1 is given by the following

Theorem 3.2. Let $M$ and $N$ be positive numbers, let $a, b$ be functions which satisfy the inequality $|a(z)+m b(z)| \geq N / M^{2}$ for all $z \in U_{1}^{n}$ and $m \geq 1$. Let $f \in H\left(U_{1}^{n}\right)$, $f(0)=0$, and suppose that

$$
\left|a(z) f(z)+b(z)[D f(z)][z]^{\prime}\right|<N / M \quad \text { for all } \quad z \in U_{1}^{n} .
$$

Then $|f(z)|<M$ in $U_{1}^{n}$.

Proof. If we suppose that the relation $|f(z)|<M$ does not hold in $U_{1}^{n}$, then, using the continuity of the Euclidean norm and the relation $f(0)=0$, we deduce that there exists a point $z_{0} \in U_{1}^{n}$ with the property

$$
M=\left|f\left(z_{0}\right)\right|=\max \left\{|f(z)|:\|z\| \leq\left\|z_{0}\right\|\right\} .
$$

Now it is sufficient to apply the conclusion of Theorem 2.1 to see that

$$
\left|a\left(z_{0}\right) f\left(z_{0}\right)+b\left(z_{0}\right)\left[D f\left(z_{0}\right)\right]\left[z_{0}\right]^{\prime}\right| \geq N / M
$$

but this is a contradiction with the hypothesis. Hence $|f(z)|<M$ for all $z \in U_{1}^{n}$.

For $a(z) \equiv 0$ in $U_{1}^{n}$, we obtain

Corollary 3.1. Let $M$ and $N$ be positive numbers and let $f$ be a holomorphic mapping on $U_{1}^{n}$ with $f(0)=0$. Suppose that $b: U_{1}^{n} \rightarrow \mathbb{C}$ is a function which satisfies the conditions

$$
\left|b(z)[D f(z)][z]^{\prime}\right|<N / M \quad \text { and } \quad|b(z)| \geq N / M^{2} \quad \text { for all } \quad z \in U_{1}^{n} .
$$

Then $|f(z)|<M$ in $U_{1}^{n}$.

Another application of Theorem 2.3 is given in 
THEOREM 3.3. Let $f \in H\left(U_{1}^{n}\right)$ and $g$ be a biholomorphic mapping on $\bar{B}_{1}^{n}$ with $g(0)=$ $f(0)$. Suppose that $\left|[D f(z)][z]^{\prime}\right|<M$ for all $z \in U_{1}^{n}$, where $M=\inf _{|\zeta|=1}\left|[D g(\zeta)]^{-1}\right|^{-1}$. Then $f \prec g$.

Proof. If this subordination does not hold, then, by Theorem 2.3, there exist the points $z_{0} \in U_{1}^{n}, \zeta_{0} \in \partial B_{1}^{n}$ and a real number $s, s \geq 1$, such that

$$
f\left(z_{0}\right)=g\left(\zeta_{0}\right) \quad \text { and } \quad\left|\left[D f\left(z_{0}\right)\right]\left[z_{0}\right]^{\prime}\right| \geq s\left|\left[D g\left(\zeta_{0}\right)\right]^{-1}\right|^{-1},
$$

so $\left|\left[D f\left(z_{0}\right)\right]\left[z_{0}\right]^{\prime}\right| \geq M$ which contradicts the hypothesis. Hence $f$ is subordinate to $g$.

\section{References}

[1] B. Chabat, Introduction à l'analyse complexe, tome 2, ed. MIR, Moscou, 1990.

[2] Sh. Gong and S. S. Miller, Partial differential subordinations and inequalities defined on complete circular domain, Comm. Partial Differential Equations, 11 (1986), 1243-1255.

[3] G. Kohr and M. Kohr-Ile, Partial differential subordinations and inequalities for holomorphic mappings of several complex variables, Ms., to appear.

[4] P. Liczberski, Jack's Lemma for holomorphic mappings, Ann. Univ. Mariae Curie-Skłodowska, Sect. A, 40 (1986), 131-139.

[5] S. S. Miller and P. T. Mocanu, Second order differential inequalities in the complex plane, J. Math. Anal. Appl., 65 (1978), 289-305.

[6] -, -, Differential subordinations and univalent functions, Michigan Math. J., 20 (1981), 157-171.

[7] - - - , The theory and applications of second order differential subordinations, Studia Univ. Babeş-Bolyai (Mathematica), 34 (1989), 13-33.

[8] T. J. Suffridge, The principle of subordination applied to functions of several variables, Pacific J. Math., 33 (1970), 241-248.

[9] - Starlike and convex maps in Banach spaces, Pacific J. Math., 46 (1973), 575-589. 